\title{
Systematic Review \\ The Orthopedic Effects of Electronic Cigarettes: A Systematic Review and Pediatric Case Series
}

\author{
Maxwell Luke Armstrong ${ }^{1, *(\mathbb{D}}$, Nicholas Smith ${ }^{1}$, Rhiannon Tracey ${ }^{2}$ and Heather Jackman ${ }^{1}$ \\ 1 Division of Orthopedic Surgery, Janeway Children's Health and Rehabilitation Centre, Memorial University of \\ Newfoundland, St. John's, NL A1B 3V6, Canada; nicksmith@munmed.ca (N.S.); hrjackman@gmail.com (H.J.) \\ 2 Division of Orthopedic Surgery, Schulich School of Medicine and Dentistry, Western University, \\ London, ON N6A 5A5, Canada; rmt224@mun.ca \\ * Correspondence: mlarmstrong@mun.ca
}

Citation: Armstrong, M.L.; Smith, N.; Tracey, R.; Jackman, H. The Orthopedic Effects of Electronic Cigarettes: A Systematic Review and Pediatric Case Series. Children 2022, 9, 62. https://doi.org/10.3390/

children 9010062

Academic Editor:

Christiaan J. A. van Bergen

Received: 9 December 2021

Accepted: 28 December 2021

Published: 4 January 2022

Publisher's Note: MDPI stays neutral with regard to jurisdictional claims in published maps and institutional affiliations.

Copyright: (C) 2022 by the authors. Licensee MDPI, Basel, Switzerland. This article is an open access article distributed under the terms and conditions of the Creative Commons Attribution (CC BY) license (https:// creativecommons.org/licenses/by/ $4.0 /)$.

\begin{abstract}
Electronic cigarette (EC) use is highly prevalent, especially in the adolescent population, where $29 \%$ of Canadian adolescents have used an EC in the past thirty days per national surveys. Our pediatric orthopedic referral centre observed a cluster of delayed unions of bone fractures in adolescents using ECs and present the case series here. We then asked whether electronic cigarettes impair bone healing or influence orthopedic outcomes. A PRISMA-compliant systematic review was carried out, which revealed no human clinical studies and a general paucity of evidence around ECs and musculoskeletal health. The existing experimental evidence relevant to orthopedics is summarized. The effect of ECs on the musculoskeletal system is poorly understood and is a target for further research.
\end{abstract}

Keywords: e-cigarette; vaping; adolescent health; pediatric orthopedics

\section{Hypothesis-Generating Cases \\ 1.1. Ankle Fracture While Roughhousing, Eighteen Weeks in a 15-Year-Old Male}

A fifteen-year-old male with no known medical history sustained a closed, minimally displaced, simple oblique trans-syndesmotic lateral malleolus fracture with no widening of the mortise. There was a remote history of occasional cigarette smoking and the patient was a current user of electronic cigarettes (EC). The fracture was reduced and splinted in a peripheral Emergency Room and referred to our centre for definitive management.

Initial treatment was circumferential casting and non-weight bearing. At one-week post-injury X-rays confirmed near-anatomic alignment. At four weeks post-injury radiographs were equivocal for callus, the patient's exam was reassuring, and a removable cast-boot was placed with progressive weight bearing. At eight weeks post-injury, there was pain at the fracture site and inability to bear full weight. The patient was counselled to discontinue EC use. Cast-boot immobilization with weight bearing as tolerated was continued. There was no clinical or radiographic change at twelve weeks post-injury (Figure 1). Delayed union was diagnosed in the absence of healing beyond the expected four to six weeks required to heal this injury [1]. At eighteen weeks post-injury the fracture was no longer symptomatic, and radiographs confirmed bony union.

\subsection{Monteggia Fracture-Dislocation on a Motorcycle, Fifteen Weeks in a 14-Year-Old Male}

A fourteen-year-old male presented with a closed, neurovascularly intact, displaced, and moderately comminuted diaphyseal ulnar fracture with associated posterolateral dislocation of the radial head, also known as a Monteggia fracture-dislocation. The injury occurred after ejection from an off-road motorcycle at moderate speed. There was an associated unstable C5/6 cervical spine fracture-dislocation and disc rupture with no 
neurological symptoms. There was no known medical history. The forearm fracturedislocation was reduced and splinted in the trauma bay by pediatric orthopedics with nearanatomic alignment. Neurosurgery colleagues managed the spine injury with emergent anterior discectomy and fusion without complication from which he recovered well with no neurologic sequelae. There was no history of cigarette smoking, but the patient was a current user of e-cigarettes.
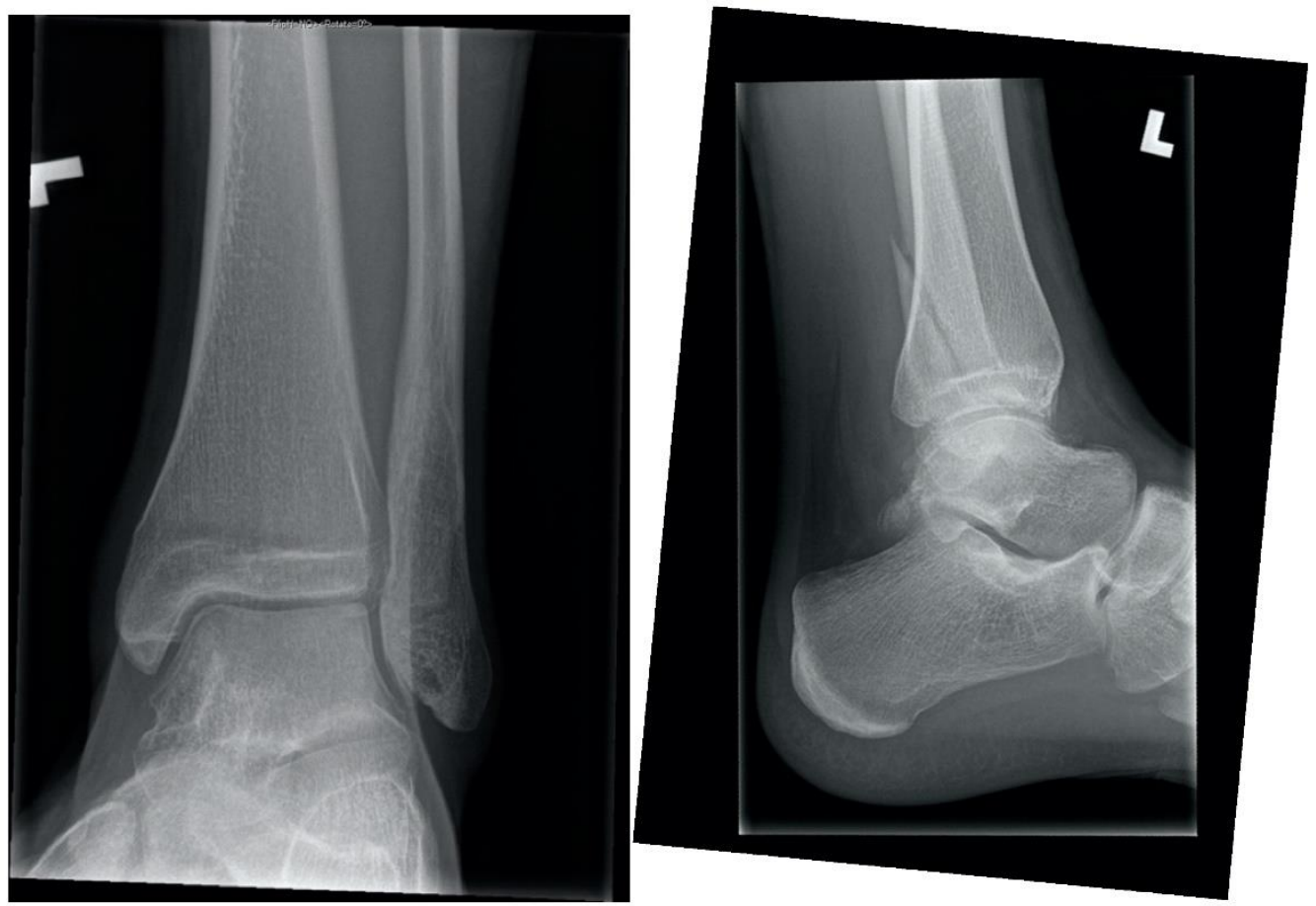

Figure 1. Twelve-week ankle radiographs demonstrating no radiographic union after fibula fracture in a pediatric electronic cigarette user.

At one-week post-injury radiographs demonstrated good alignment of the fracture and radio-capitellar joint. A long-arm circumferential cast was placed. At three weeks postinjury radiographs again demonstrated acceptable alignment and casting was continued. At seven weeks post-injury, there was no radiographic evidence of healing, and a removable cast was prescribed with instructions for twice daily non-weightbearing range of motion exercises. The patient was counselled to discontinue EC use. At eleven weeks post-injury there was continued pain at the fracture site with no radiographic evidence of healing (Figure 2). Delayed union was diagnosed as the expected healing time of a closed pediatric forearm fracture is approximately 5.5 weeks [1]. Fifteen weeks post-injury the patient had symptomatic improvement with resolution of fracture-site tenderness and radiographs showed evidence of bony union.

\subsection{Forearm Fracture on a Trampoline, Eighteen Weeks in a 15-Year-Old Male}

A fifteen-year-old male sustained closed, neurovascularly intact, displaced fractures of the radial and ulnar diaphyses with comminution of the radius. The mechanism of injury was a fall on an outstretched hand on a trampoline. The patient had no systemic comorbidity and no history of smoking but was a current user of electronic cigarettes. The fracture was splinted in a peripheral Emergency Room and was referred to our centre. The following day, the fractures were definitively treated without complication by open reduction internal fixation (ORIF) with plates and screws. The simple ulnar fracture was plated in compression mode, and the mildly comminuted radial fracture was approximated and plated in bridge mode. 


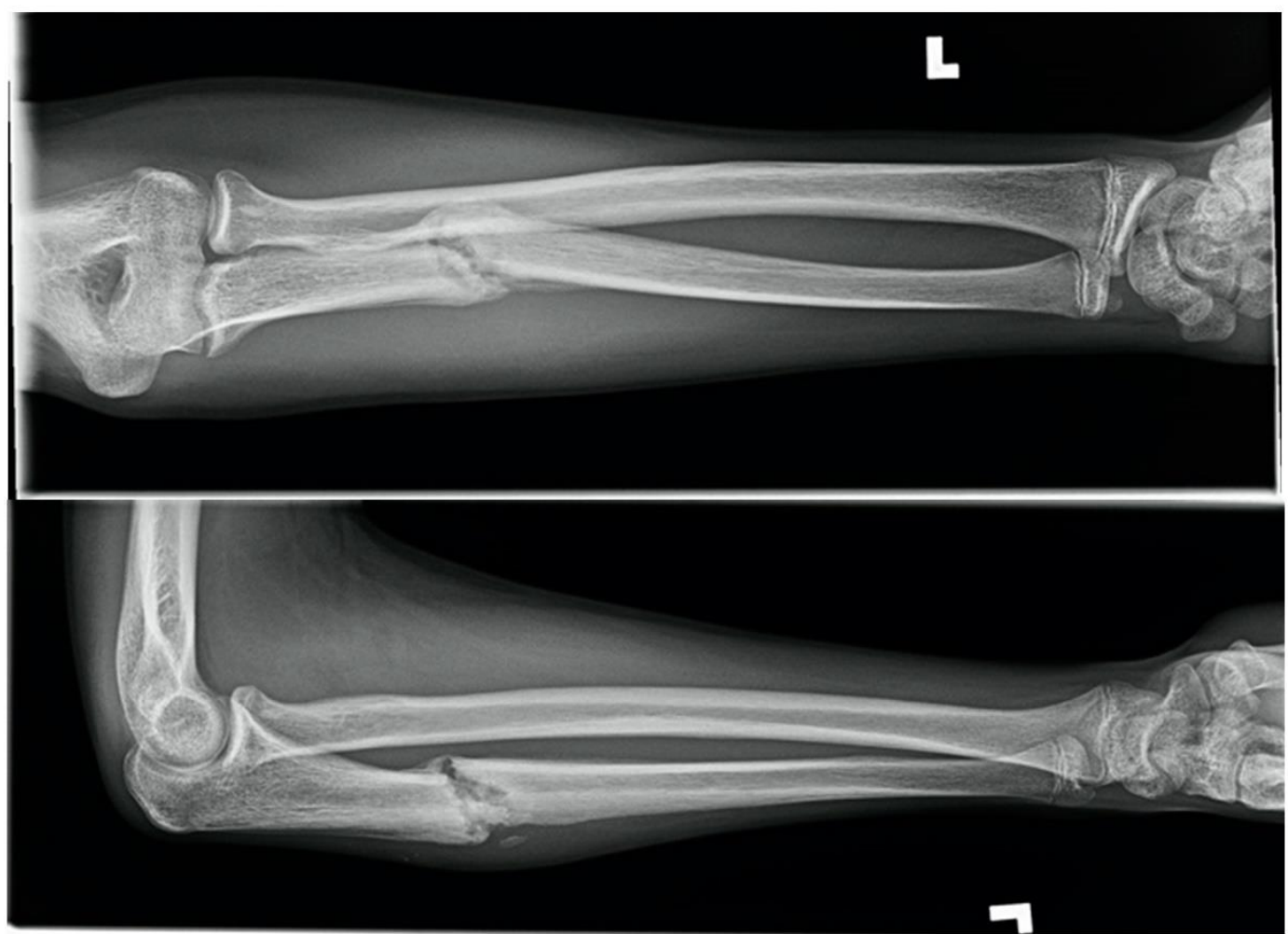

Figure 2. Eleven-week forearm radiographs demonstrating no union after Monteggia fracturedislocation in a pediatric EC user.

Five weeks after operative treatment splint immobilization was discontinued, and a functional brace was applied. Eight weeks after ORIF, radiographs demonstrated bony union of the ulna but no union of the radius fracture despite anatomic alignment. The patient was counselled to discontinue EC use. Again, at twelve weeks, there was no union of the radius fracture and the patient had ongoing symptoms (Figure 3). Delayed union was diagnosed. Eighteen weeks after ORIF the patient had clinically improved, radiographs demonstrated bony union, and treatment was discontinued.

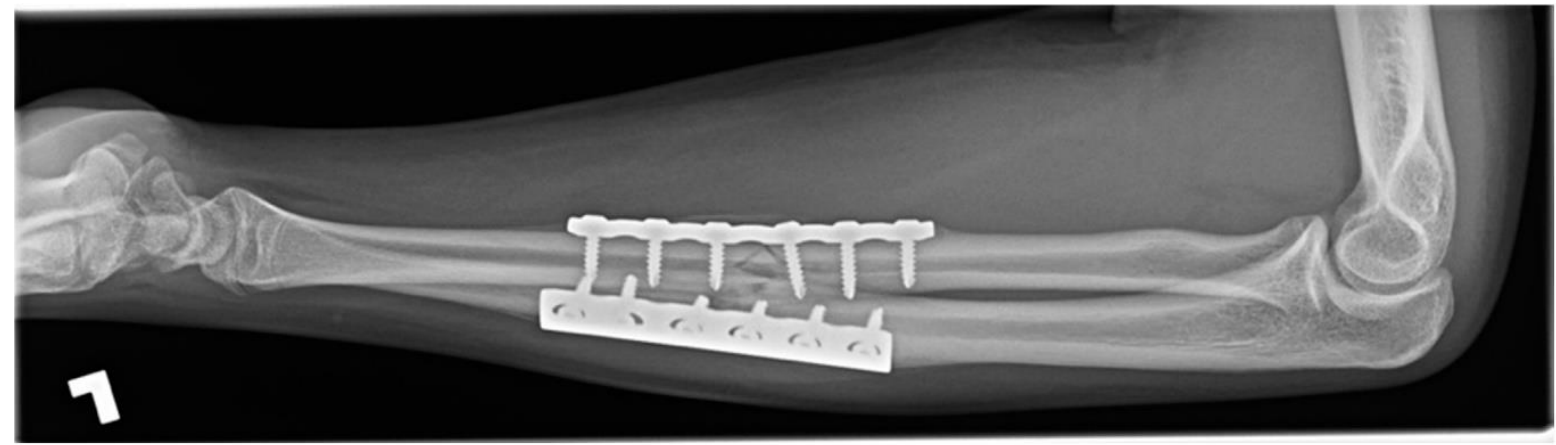

Figure 3. Twelve-week radiograph of an operatively treated both-bones forearm fracture in a pediatric electronic cigarette user. The ulna is healed, but the radius is not.

\section{Background and Rationale for Review}

Impaired bone healing is rare in the pediatric population. A large Scottish study found 422 fracture non-unions in 161,100 fractures diagnosed in patients aged 0-18 over five years, for an overall incidence of $0.2-0.35 \%$ per fracture [2]. Risk factors for impaired bone healing in pediatric patients include specific fracture characteristics, local bone biology, systemic 
host health, increasing age, and orthopedic treatment tactics. Complications of impaired bone healing may include pain, deformity, reduced mobility, and unplanned surgery [1].

During a review of the fractures treated at our pediatric orthopedic referral centre, the above cases were noted as a cluster of difficult-to-heal fractures. Retrospectively, we observed the common exposure of e-cigarette use. During the ten months in 2018-2019 when these cases were managed, 330 fractures in patients aged 12 to 18 were treated at our centre. Three of these 330 fractures had delayed union, which are the three presented above. This led us to pose the question: Do electronic cigarettes affect bone healing?

These cases are presented without any inference of an association between ECs and bone healing and serve only as a hypothesis generator. The impaired bone healing in our three cases may be attributable to a variety of factors. For example, in Case 1, patient adherence to removable cast-boot immobilization of the fibula fracture is unknown. In Case 2, a higher energy mechanism, the biology of the ulnar diaphysis, and nonoperative treatment tactics may have contributed to impaired healing. An iatrogenic fracture gap created by a bridging plate and screw fixation of a comminuted radial shaft fracture in Case 3 could be a treatment-related non-union risk factor.

Electronic cigarettes (ECs) are pocket-sized devices that combine heat and the user's inhalation to vaporize a solution to produce a cloud of vapor that mimics the experience of conventional cigarette smoking, often called 'vaping'. The solutions are proprietary but are usually composed of water, propylene glycol, and glycerine, as well as additives of flavorings and nicotine in varying concentrations [3]. A proposed danger of ECs is the ability to achieve high nicotine doses by selecting a solution with high nicotine content and vaping more frequently [3]. The long-term health effects of these products are unknown, as are the actual chemicals delivered to the bloodstream in vivo after passing the solution over a superheated coil. The characterization of the 'E-cigarette or vaping product useassociated lung injury' (EVALI) has been a recent area of intense research focus and media scrutiny [4,5]. Significant attention has also duly been given to the diagnosis and treatment of facial blast injuries from exploding devices [6].

The popularity of ECs amongst Canadian adolescents is alarming [4]. The Canadian Student Tobacco, Alcohol, and Drugs Survey (CSTADS) 2018-2019 demonstrated that 29\% of students in high school were current users and had vaped in the past 30 days, a rapid increase from prior years. Daily or occasional cigarette smoking had a 3\% prevalence, which was stable [7].

Complicating the issue is the marketing of ECs, which usually suggests that they are a healthier, 'cleaner' alternative to cigarettes. This appeals to consumers who perceive a healthier option with a similar experience to smoking. ECs are marketed to adults as a smoking cessation option. A range of vapour flavours and modern device styling with USB charging have likely contributed to the concerning prevalence of use in the pediatric population [7].

It is tempting to equate the health effects of conventional cigarettes and ECs as they both deliver nicotine via inhalation and the user experience is similar. However, they are chemically dissimilar products except for nicotine. While the negative effect of tobacco smoking on bone healing is unequivocal, the contribution to this effect from the chemical nicotine specifically is still under investigation $[8,9]$.

This study asked whether electronic cigarettes had a deleterious effect on bone healing and, more broadly, on orthopedic outcomes. We conducted a PRISMA-compliant systematic review and present the results below.

\section{Systematic Review}

\subsection{Eligibility Criteria}

All publications with potential relevance to the operative or non-operative practice of orthopedics in English or French were examined (no results were found in other languages). For completeness, human, animal, and experimental studies were considered from all levels of evidence. 


\subsection{Search Strategy and Sources}

In March 2021, the PubMed, EMBASE, and Cochrane databases were searched for relevant publications. Each of these databases were interrogated with the basic input, ((e-cigarette) OR (vaping)) AND ((orthopedics) OR (surgery)). The extended search terms from PubMed are displayed in Table 1 as an example and no limits or filters were used. To search the grey literature, the OpenGrey database (www.opengrey.eu, accessed on 22 March 2021) was searched with the same terms. This strategy yielded 608 papers for consideration after removal of duplicates.

Table 1. Detailed searching strategy.

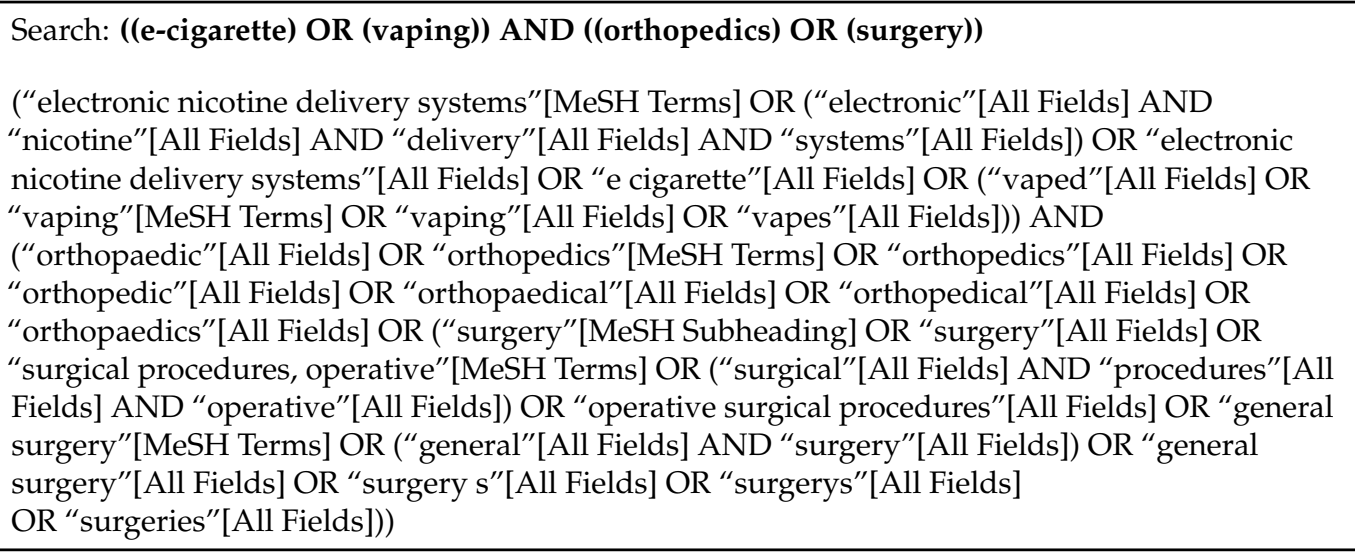

\subsection{Study Selection and Data Colletion Process}

Upon initial review of the results, it was obvious that our primary question of the effects of e-cigarettes on bone healing would not be answered by the current literature. There were no human studies of bone healing in EC users. We pursued our broader goal of collecting any literature pertaining to the effect of e-cigarettes on orthopedic outcomes. The corresponding author (M.L.A.) sorted and screened the initial 608 records for potential relevance to orthopedics based on the title and abstract. Records with titles indicating relevance exclusively to a non-orthopedic topic (i.e., smoking cessation, economics, sociology) were excluded. All potentially relevant abstracts were reviewed and excluded if there was no pertinence to the musculoskeletal system and ECs or EC vapour.

Thirty-five potentially relevant records were then retrieved for manuscript assessment. No automation tools were used. The title, abstract, and manuscript were read and assessed for pertinence to orthopedics. Further exclusions were made on the basis of no relevance to orthopedics $(n=11)$, no relevance to electronic cigarettes $(n=1)$, and for a focus only on smoking cessation $(n=2)$. Publications were grouped for their topic/purpose and quality of evidence. The systematic review workflow is summarized in Figure 4.

This review protocol was submitted for registration on the International Prospective Register of Systematic Reviews (PROSPERO identification number: 299563). 


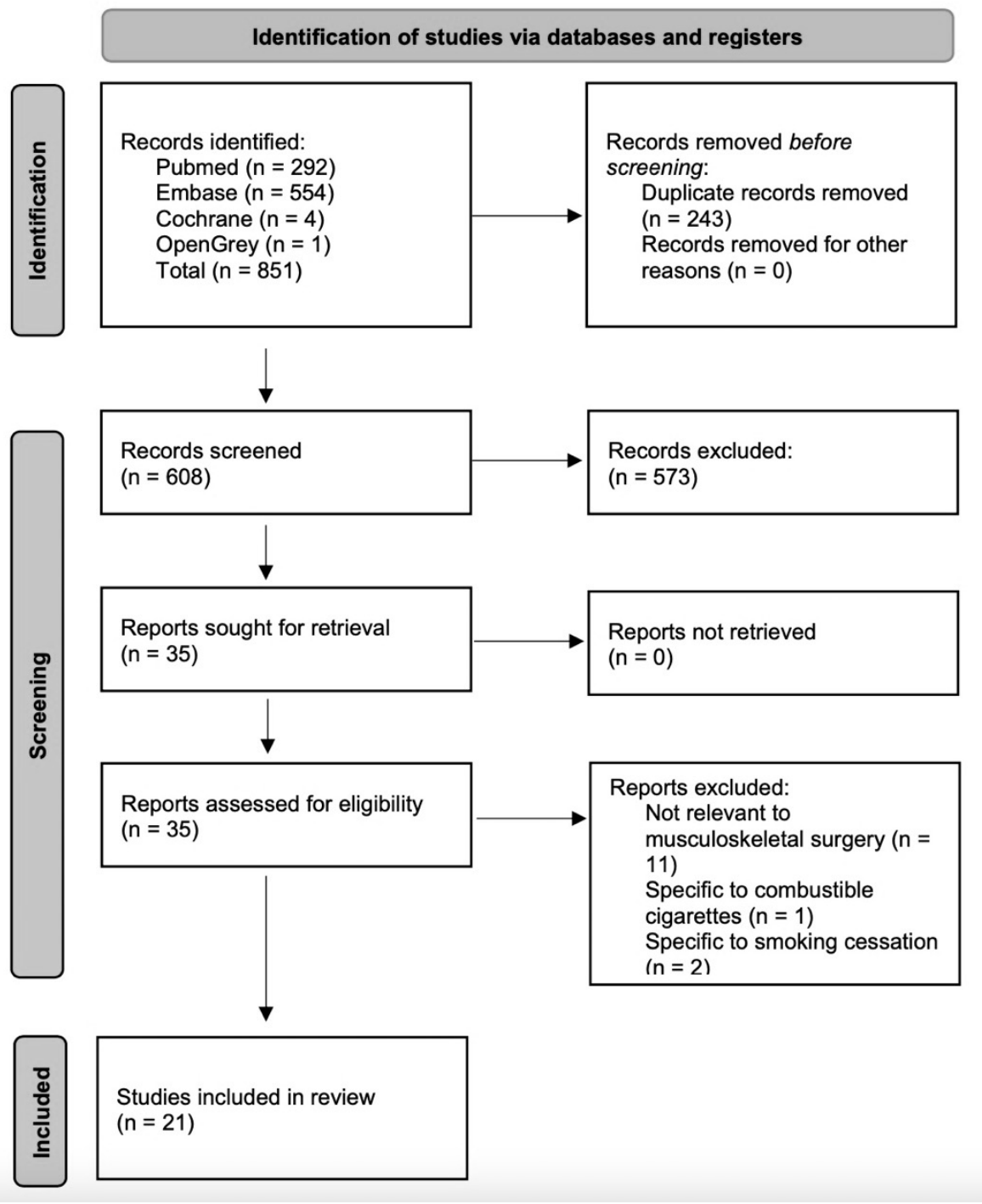

Figure 4. Systematic review flowchart.

\section{Results}

No Level 1 studies were found. No human clinical studies were found. Only six studies were found that were experimental, which are summarized in Table 2. In summarizing these studies, they clustered around three subtopics-basic science, musculoskeletal health, and wound healing. Data extraction was not applicable as no relevant human clinical studies on e-cigarettes were found.

Most publications were non-systematic reviews, briefs, and opinion letters. These are not discussed here but are cited in the references list per Table 2.

Table 2. Summary table of included publications.

Level 1: Meta-analyses and randomized controlled trials

Nil

Level 2: Unrandomized trials, cohort studies, scientific experiments

Basic science $\quad$ Pywell et al. [10] $\begin{aligned} & \text { Nonclinical human } \\ & \text { experiment }\end{aligned}$

Romagna et al. [11] Cell line experiment


Table 2. Cont.

\begin{tabular}{lll}
\hline MSK health & Kennedy et al. [12] & Animal experiment \\
\hline Dermal healing & Reumann et al. [13] & Animal experiment \\
\hline & Rau et al. [14] & Animal experiment \\
\hline Level 3: Expert opinion, briefs, reports & Troiano et al. [15] & Animal experiment \\
\hline Non-systematic review, orthopedic & Publications [16-18] & \\
\hline Non-systematic review, surgery, and wounds & Publications [19-22] & \\
\hline Briefs and opinion & Publications [23-30] & \\
\hline
\end{tabular}

\section{Discussion}

Our systematic review highlights the paucity of evidence about the effect of ECs on orthopedic surgery and the musculoskeletal system in general. The papers that were found pertained to basic science, musculoskeletal health, and wound healing.

Pywell et al. published a human experiment on 15 subjects that demonstrated a reduction in hand microcirculation after inhaling a $24 \mathrm{mg}$ nicotine e-cigarette but not after a $0 \mathrm{mg}$ ecigarette. They concluded the consumption of nicotine causes vasoconstriction [10]. Romagna et al. exposed fibroblast cell cultures to liquified solutions of EC vapor and combustible cigarette smoke and found the liquified smoke to be significantly more cytotoxic [11].

Kennedy et al. undertook an animal experiment with rats exposed to two cigarettes daily or the nicotine equivalent of electronic cigarette vapour for four weeks. The rats had Achilles tendon transection and repair performed and then the tensile strength of the repair was measured two weeks later. The EC cohort had a significantly lower load-tofailure versus the cigarette and control cohorts, which were not significantly different [12]. Reumann et al. exposed mice to EC vapour or the nicotine equivalent of cigarette smoke daily for six months and then quantified bone strength parameters. In this study, the cigarette smoke significantly decreased bone density and bending strength compared to EC vapour and the control group, which were not significantly different [13].

In 2019, Rau et al. raised and then repaired skin flaps on a rat model exposed to low-dose EC vapour, high-dose EC vapour, or combustible cigarettes for four weeks. Five weeks after surgery flap survival was significantly decreased in the exposure groups versus the control group, but not within the three exposure groups [14]. This rat experiment was essentially repeated by Troiano et al., who also found no significant difference in flap necrosis between EC vapour- or cigarette-exposed rats but did find a significant increase in necrosis in these exposed groups versus the control [15].

Other groups have published non-systematic reviews and expert briefs of the topic, including Amaro et al. in JBJS Reviews in 2019. They compared and contrasted ECs with combustible cigarettes and overviewed the orthopedic issues [17]. Fiani et al. similarly overviewed the topic with a focus on the spine and molecular toxicology [18]. Nicholson et al. recently published an expert brief on the musculoskeletal biochemistry of vaping in Bone and Joint Research [16].

This systematic review is the most current collection of knowledge about the orthopedic effects of electronic cigarettes. This review is severely limited in its ability to draw any clinically relevant conclusions about the orthopedic effects of electronic cigarettes based on the current body of evidence. A further limitation is that a single researcher reviewed the searched publications for suitability, which may introduce bias in judgement or clerical error.

Vaping is highly prevalent, especially amongst adolescents, and its orthopedic effects are poorly understood. We presented three cases as a clinical observation that served as a hypothesis generator. We conducted a PRISMA-compliant systematic review that revealed a scarcity of evidence about vaping and musculoskeletal health. This paper serves 
as a starting point and a call for high-quality investigation into the orthopedic effects of electronic cigarettes.

Author Contributions: Conceptualization, H.J. and N.S.; methodology, M.L.A. and R.T.; investigation, M.L.A.; resources, H.J.; data curation, M.L.A.; writing-original draft preparation, M.L.A. and R.T.; writing-review and editing, M.L.A., N.S. and H.J.; supervision, H.J.; project administration, M.L.A. All authors have read and agreed to the published version of the manuscript.

Funding: This research received no external funding.

Institutional Review Board Statement: The study was conducted according to the guidelines of the Declaration of Helsinki and approved by the Health Research Ethics Board of Memorial University of Newfoundland (HREB \# 2019.191).

Informed Consent Statement: Informed consent was obtained from all subjects involved in the study.

Data Availability Statement: No new data were created or analyzed in this study. Data sharing is not applicable to this article.

Conflicts of Interest: The authors declare no conflict of interest.

\section{References}

1. Waters, P.M.; Skaggs, D.L.; Flynn, J.M. (Eds.) Rockwood \& Wilkins' Fractures in Children, 9th ed.; Lippincott Williams \& Wilkins: Philadelphia, PA, USA, 2019.

2. Mills, L.A.; Simpson, A.H. The risk of non-union per fracture in children. J. Child. Orthop. 2013, 7, 317-322. [CrossRef] [PubMed]

3. Breland, A.B.; Spindle, T.; Weaver, M.; Eissenberg, T. Science and Electronic Cigarettes. J. Addict. Med. 2014, 8, 223-233. [CrossRef] [PubMed]

4. Perikleous, E.P.; Steiropoulos, P.; Paraskakis, E.; Constantinidis, T.C.; Nena, E. E-Cigarette Use Among Adolescents: An Overview of the Literature and Future Perspectives. Front. Public Health 2018, 6, 86. [CrossRef]

5. Bold, K.W.; Kong, G.; Camenga, D.R.; Simon, P.; Cavallo, D.A.; Morean, M.E.; Krishnan-Sarin, S. Trajectories of E-Cigarette and Conventional Cigarette Use Among Youth. Pediatrics 2017, 141, e20171832. [CrossRef] [PubMed]

6. Serror, K.; Chaouat, M.; Legrand, M.M.; Depret, F.; Haddad, J.; Malca, N.; Mimoun, M.; Boccara, D. Burns caused by electronic vaping devices (e-cigarettes): A new classification proposal based on mechanisms. Burns 2018, 44, 544-548. [CrossRef]

7. Government of Canada. Summary of Results for the Canadian Student Tobacco, Alcohol and Drugs Survey 2016-17; Statistics Canada: Ottawa, ON, Canada, 2018.

8. Scolaro, J.A.; Schenker, M.L.; Yannascoli, S.M.; Baldwin, K.; Mehta, S.; Ahn, J. Cigarette Smoking Increases Complications Following Fracture. J. Bone Jt. Surg. 2014, 96, 674-681. [CrossRef]

9. Argintar, E.; Triantafillou, K.; Delahay, J.; Wiesel, B. The Musculoskeletal Effects of Perioperative Smoking. J. Am. Acad. Orthop. Surg. 2012, 20, 359-363. [CrossRef]

10. Pywell, M.J.; Wordsworth, M.; Kwasnicki, R.M.; Chadha, P.; Hettiaratchy, S.; Halsey, T. The Effect of Electronic Cigarettes on Hand Microcirculation. J. Hand Surg. 2018, 43, 432-438. [CrossRef]

11. Romagna, G.; Allifranchini, E.; Bocchietto, E.; Todeschi, S.; Esposito, M.; Farsalinos, K. Cytotoxicity evaluation of electronic cigarette vapor extract on cultured mammalian fibroblasts (ClearStream-LIFE): Comparison with tobacco cigarette smoke extract. Inhal. Toxicol. 2013, 25, 354-361. [CrossRef]

12. Kennedy, P.; Saloky, K.; Yadavalli, A.; Barlow, E.; Aynardi, M.; Garner, M.; Bible, J.; Lewis, G.; Dhawan, A. Comparison of Achilles Tendon Healing After Exposure to Combusted Tobacco, Vaping, and Control in a Rat Model. Orthop. J. Sports Med. 2019, 7, 2325967119S00328. [CrossRef]

13. Reumann, M.K.; Schaefer, J.; Titz, B.; Aspera-Werz, R.H.; Wong, E.T.; Szostak, J.; Häussling, V.; Ehnert, S.; Leroy, P.; Tan, W.T.; et al. E-vapor aerosols do not compromise bone integrity relative to cigarette smoke after 6-month inhalation in an ApoE-/-mouse model. Arch. Toxicol. 2020, 94, 2163-2177. [CrossRef]

14. Rau, A.S.; Reinikovaite, V.; Schmidt, E.; Taraseviciene-Stewart, L.; Deleyiannis, F.W.-B. Electronic Cigarettes Are as Toxic to Skin Flap Survival as Tobacco Cigarettes. Ann. Plast. Surg. 2017, 79, 86-91. [CrossRef]

15. Troiano, C.; Jaleel, Z.; Spiegel, J.H. Association of Electronic Cigarette Vaping and Cigarette Smoking With Decreased Random Flap Viability in Rats. JAMA Facial Plast. Surg. 2019, 21, 5-10. [CrossRef]

16. Nicholson, T.; Scott, A.; Ede, M.N.; Jones, S.W. Do E-cigarettes and vaping have a lower risk of osteoporosis, nonunion, and infection than tobacco smoking? Bone Jt. Res. 2021, 10, 188-191. [CrossRef] [PubMed]

17. Amaro, E.J.; Shepard, N.; Moss, L.; Karamitopoulos, M.; Lajam, C. Vaping and Orthopaedic Surgery: A Review of Current Knowledge. JBJS Rev. 2019, 7, e5. [CrossRef]

18. Fiani, B.; Noblett, C.; Nanney, J.M.; Gautam, N.; Pennington, E.; Doan, T.; Nikolaidis, D. The Impact of "Vaping" Electronic Cigarettes on Spine Health. Cureus J. Med. Sci. 2020, 12, e8907. [CrossRef] 
19. Fracol, M.; Dorfman, R.; Janes, L.; Kulkarni, S.; Bethke, K.; Hansen, N.; Kim, J. The Surgical Impact of E-Cigarettes: A Case Report and Review of the Current Literature. Arch. Plast. Surg. 2017, 44, 477-481. [CrossRef] [PubMed]

20. Lemay, F.; Baker, P.; McRobbie, H. Electronic cigarettes: A narrative review of the implications for the pediatric anesthesiologist. Pediatr. Anesthesia 2020, 30, 653-659. [CrossRef] [PubMed]

21. Cope, G. E-cigarettes and wound healing. Wounds UK 2020, 16, 653-659.

22. Taub, P.J.; Matarasso, A. E-Cigarettes and Potential Implications for Plastic Surgery. Plast. Reconstr. Surg. 2016, 138, 1059e-1066e. [CrossRef]

23. Farsalinos, K.; Voudris, V. It is preferable for surgical patients to use e-cigarettes rather than smoke cigarettes. BMJ 2014, 348, g1961. [CrossRef]

24. Agochukwu, N.; Liau, J.Y. Debunking the myth of e-cigarettes: A case of free flap compromise due to e-cigarette use within the first 24 hours. J. Plast. Reconstr. Ae. Surg. 2018, 71, 451-453. [CrossRef]

25. Davies, C.S.; Ismail, A. Nicotine has deleterious effects on wound healing through increased vasoconstriction. BMJ 2016, 353, i2709. [CrossRef]

26. Debusk, W.T.; Debusk, W.; Lassig, A.A.D. Implications of electronic cigarettes on postoperative wound healing. Otolaryngol. Head Neck Surg. 2020, 163, P46-P47. [CrossRef]

27. Desai, S.C. Is e-Cigarette Vaping a New Clinical Challenge to Wound Healing? JAMA Facial Plast. Surg. 2019, 21, 10-11. [CrossRef] [PubMed]

28. Holmes, W.J.M.; Southern, S.J. Detrimental effects of e-cigarettes on surgical outcomes. BMJ 2014, 348, g1156. [CrossRef] [PubMed]

29. Jeevaratnam, J.; Vijayan, R.; Blackburn, A. Hidden risks of electronic cigarettes to patients undergoing plastic surgery. Eur. J. Plast. Surg. 2014, 37, 457-458. [CrossRef]

30. Patients Should Stop e-Cigarette Use Before Plastic Surgery. J. Calif. Dent. Assoc. 2017, 45, 69. [PubMed] 